\title{
Data Preparation for Validation Study of Hazus Canada Flood Model
}

I3R2 Conference

May 20, 2014

Perdue University
H. McGrath (UNB) E. Stefanakis (UNB) M. Nastev (NRCan) M. McCarthy (PSC) 


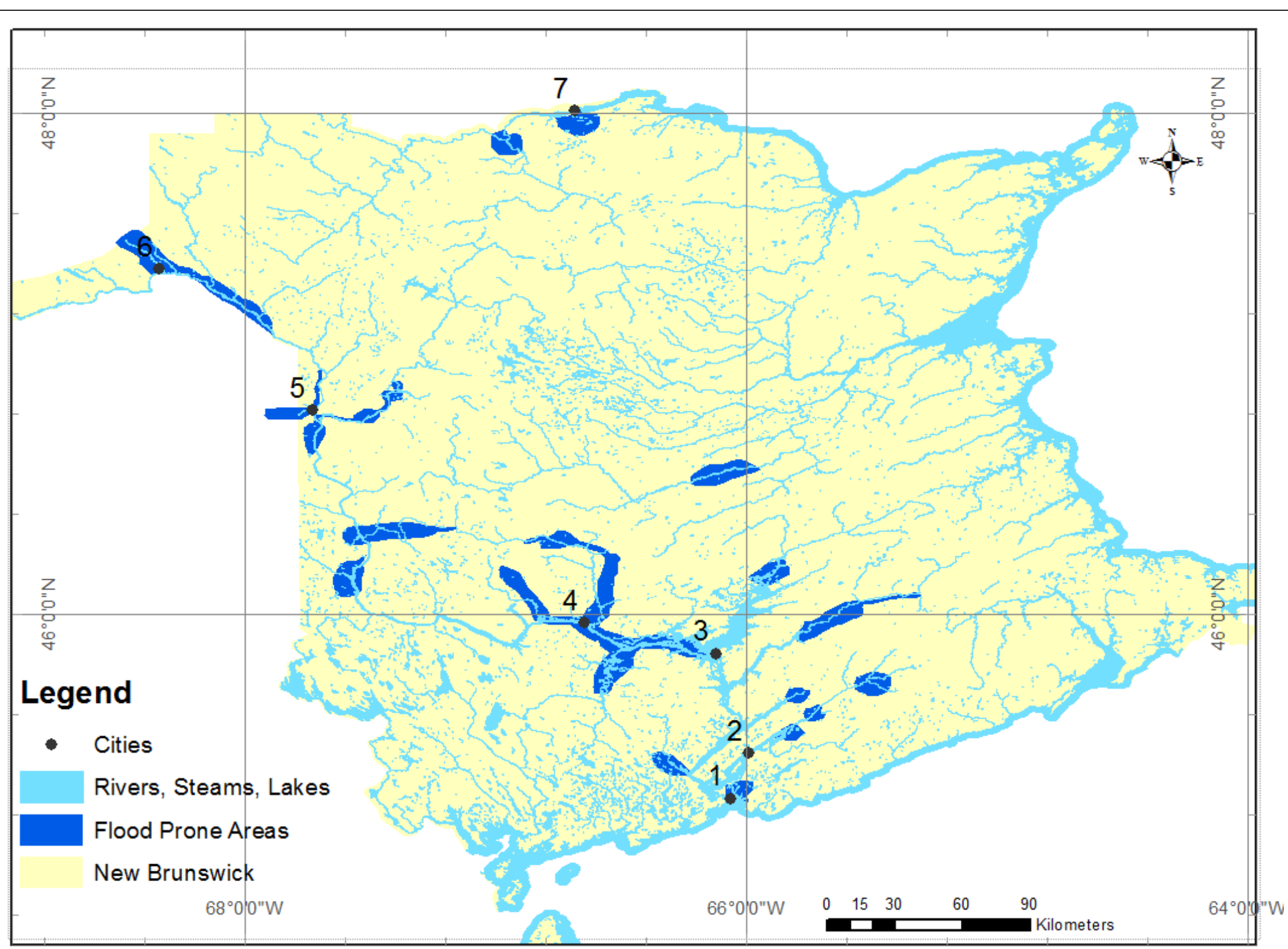

New Brunswick

$68^{\circ} 0 \prime 0^{\prime \prime} \mathrm{W}$

2. Quispamsis

3. Jemseg

4. Fredericton 5. Perth-Andover 6. Edmundston

7. Campbelton 


\section{Flood History in NB}

- 1887 - 1971 damages

- > \$1 million

- 1971 - 1976, damages

- \$17 million

- Since 1900s

- > \$133 million

- 2014 flood, in Sussex, NB

- estimates: \$12million

- 1960-1970s, settlement along Saint John River floodplain

- Increased by $30 \%$

\begin{tabular}{|l|c|c|} 
Candidate City & $\begin{array}{c}\text { \# floods } \\
\text { (last } \\
10 y r s)\end{array}$ & $\begin{array}{c}\text { Damage } \\
\text { amount } \\
\text { (thousand } \$ \text { ) }\end{array}$ \\
\hline Edmundston & 5 & $\$ 23,288$ \\
\hline Perth-Andover & 2 & $\$ 54,483$ \\
\hline Fredericton & 8 & $\$ 43,068$ \\
\hline $\begin{array}{l}\text { Hartland/ } \\
\text { Somverville }\end{array}$ & 6 & $\$ 29,483$ \\
\hline Simonds & 3 & $\$ 23,288$ \\
\hline Saint John & 3 & $\$ 16,000$ \\
\hline Quispamsis & 3 & $\$ 23,288$ \\
\hline Clair/Fort Kent & 2 & $\$ 23,288$ \\
\hline Woodstock & 2 & $\$ 42,718$ \\
\hline Maugerville & 4 & $\$ 29,483$ \\
\hline Jemseg & 5 & $\$ 28,888$ \\
\hline
\end{tabular}




\section{Adaptation of Hazus for Canadian}

Settings

- Adapting Hazus -MH (developed by

FEMA)

- Earthquake model available

- Flood model (Summer 2014)

- Scientifically calculate:

- Extent of areas vulnerable to flood hazard

- Calculate the potential damage to the exposed infrastructure

- Calculate repair and replacement costs in the risk areas

- (FEMA, 2009)

- Hazus is a mitigation tool!

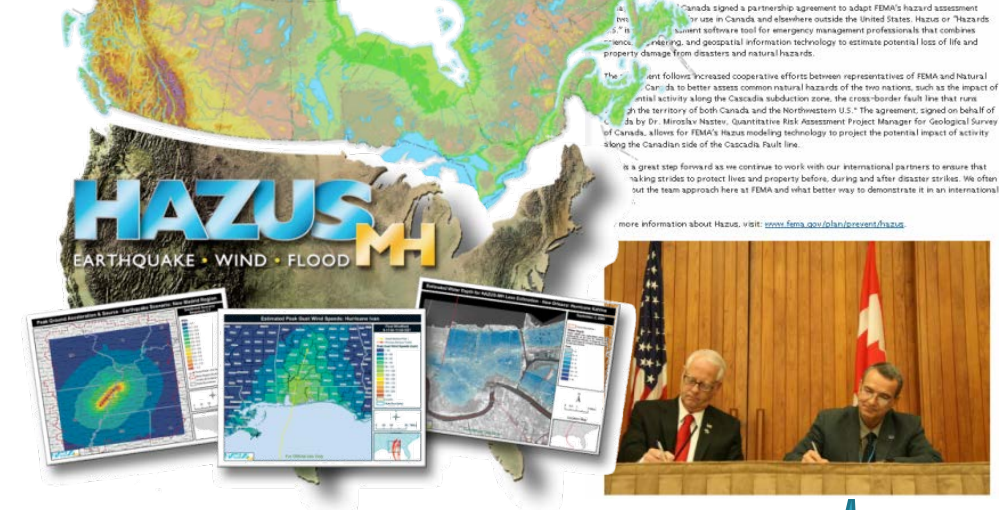




\section{Community Selected}

- Fredericton, NB

- Capital of the province

- Open data policy

- Aging Mactaquac Dam

- Flood history

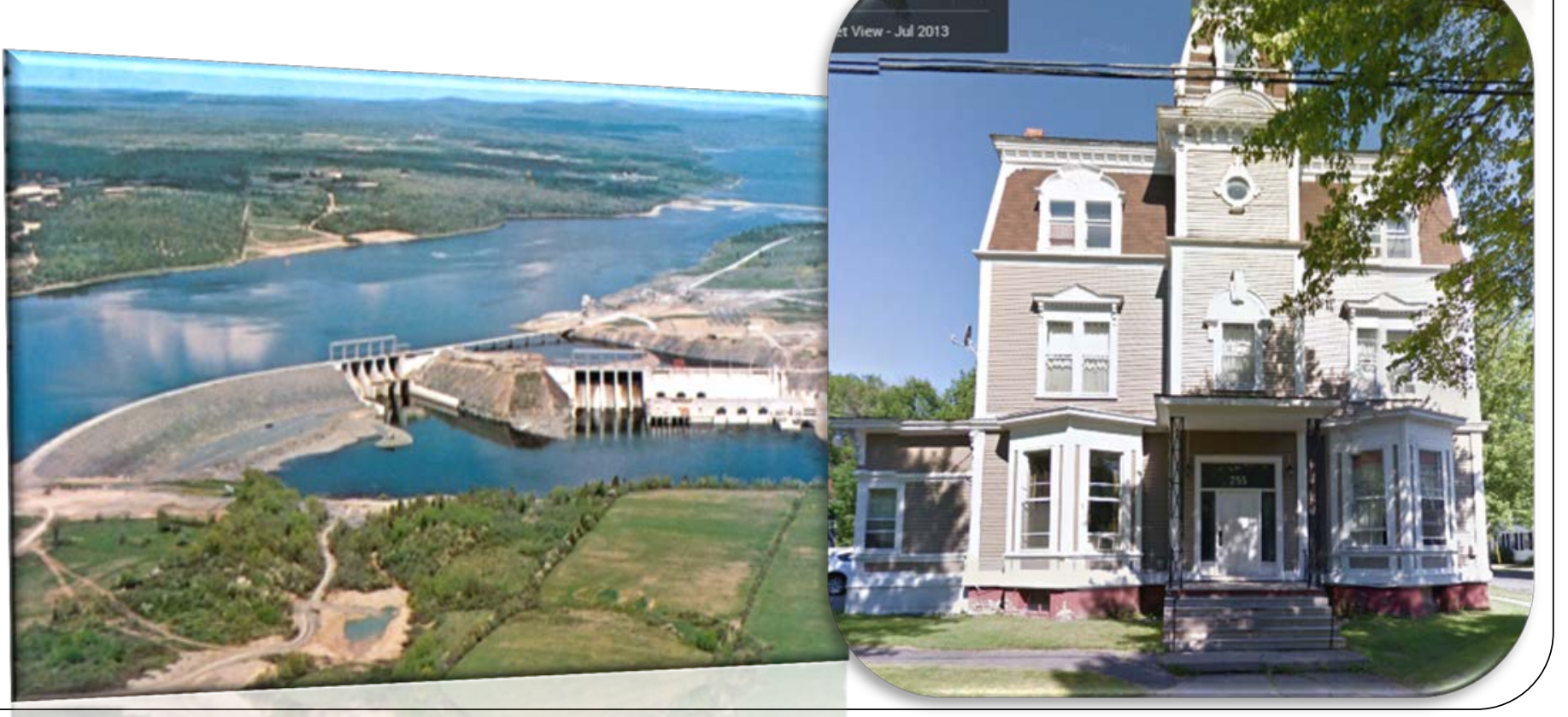




\section{Fredericton, NB}

Population: $\sim 56,000$

$\sim 22,000$ households

Mix of Public and Private infrastructure

Historic buildings: dating back to late 1700s

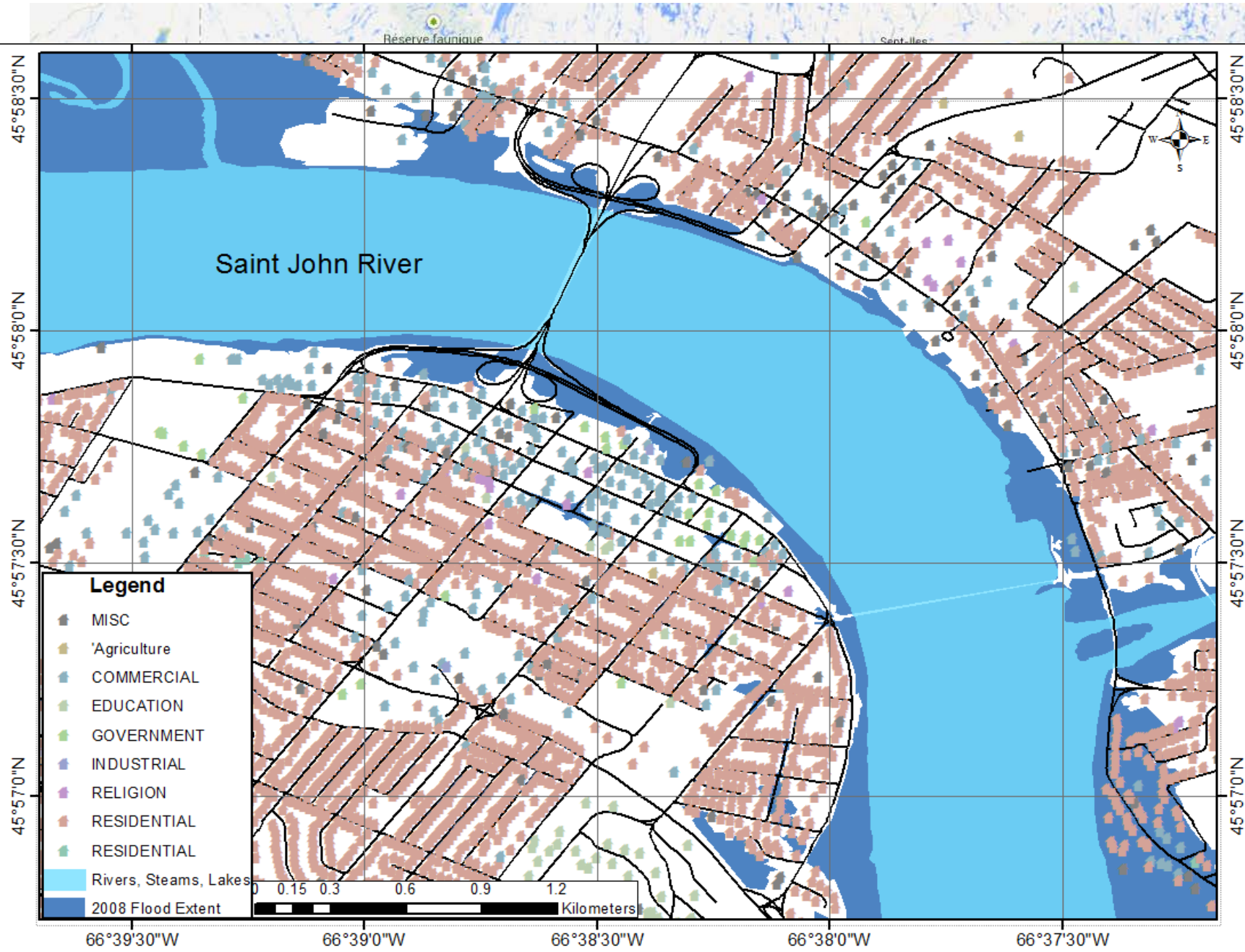




\section{Hazus Risk Assessment Process}

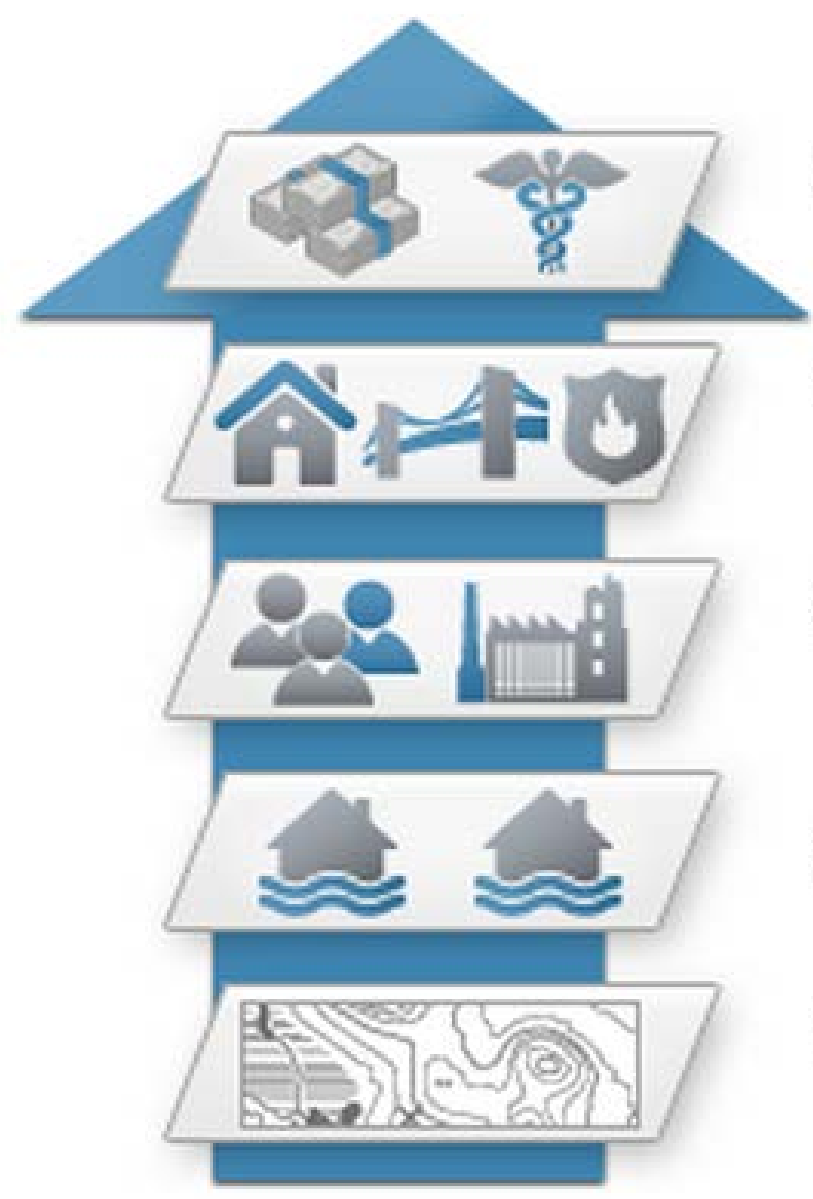

5. Produces maps, tables, and reports

4. Analyzes social and economic impacts

3. Considers what is at risk

2. Identifies hazard

1. Analyzes physical landscape 


\section{HAZARD}

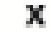

\section{INVENTORY}

\begin{tabular}{|c|}
\hline Flood \\
\hline $\begin{array}{l}\text { Riverine } \\
\text { (default } \& \\
\text { user supplied) }\end{array}$ \\
\hline $\begin{array}{l}\text { Coastal } \\
\text { (default \& } \\
\text { user supplied) }\end{array}$ \\
\hline $\begin{array}{l}\text { Riverine and } \\
\text { coastal } \\
\text { (default } \& \\
\text { user supplied) }\end{array}$ \\
\hline $\begin{array}{l}\text { Coastal surge } \\
\text { (default } \& \\
\text { user supplied) }\end{array}$ \\
\hline
\end{tabular}

stock

\section{Essential}

user supplied)

\section{Demography}

\section{General building}

residential, commercial industrial, agricultural,

$\rightarrow$ religious, government, and educational buildings

facilities (default \&

$\rightarrow$ hospitals, police stations,

fire stations and schools

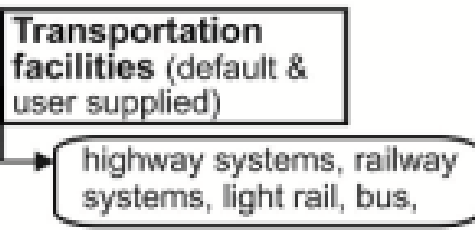

Agriculture (default \& user supplied)

Vehicles (default \& user supplied)
X

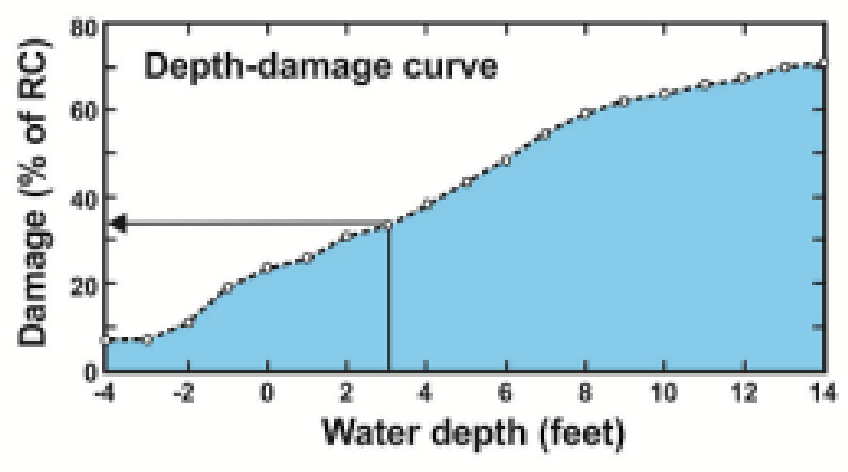

$=$ CONSEQURNCES

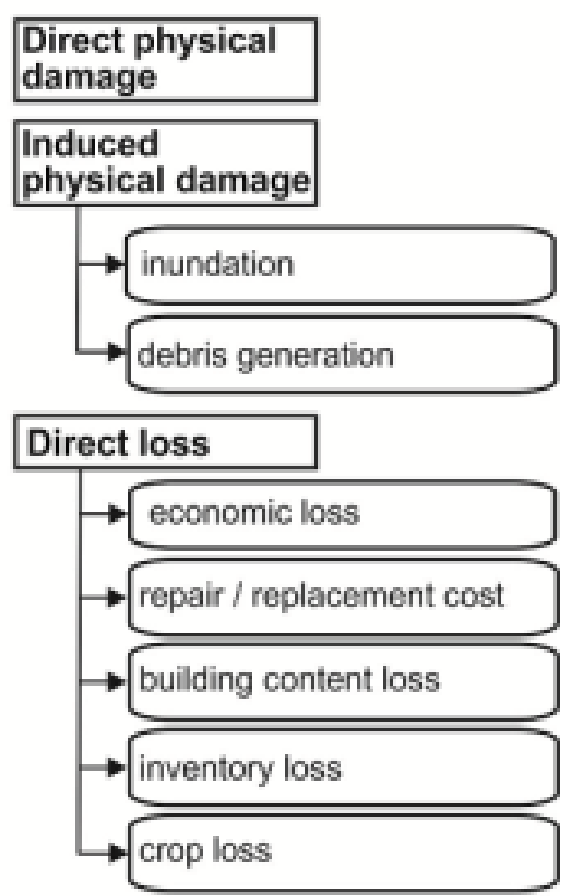

\section{Social loss}

displaced households,

short-term shelter needs

\section{Indirect loss}

supply shortages, sales decline, opportunity costs, economic loss 
Department / Municipality

City of Fredericton

City of Fredericton

City of Fredericton

City of Fredericton

City of Fredericton

City of Fredericton

City of Fredericton

New Brunswick Department of

Environment

New Brunswick Department of

Education and Early Childhood

Development

Public Safety New Brunswick

Public Safety New Brunswick

Public Safety New Brunswick

Service New Brunswick

SNB - GeoNB

SNB - GeoNB

SNB - GeoNB

SNB - GeoNB

SNB - GeoNB

\section{Available Datasets}

Building Footprints

Fire Stations

Police Offices

Parking Facilities

Street Centreline

Wastewater, potable water

LiDAR data (2008)

Flood depth grids

Public Schools

Local infrastructure

Cell Towers

Disaster financial assistance

Property Assessment

Digital Property Maps (DPM)

Flood risk areas \& historical floods

National Railway Network

Pipelines

Road Network 


\section{Building Datasets}

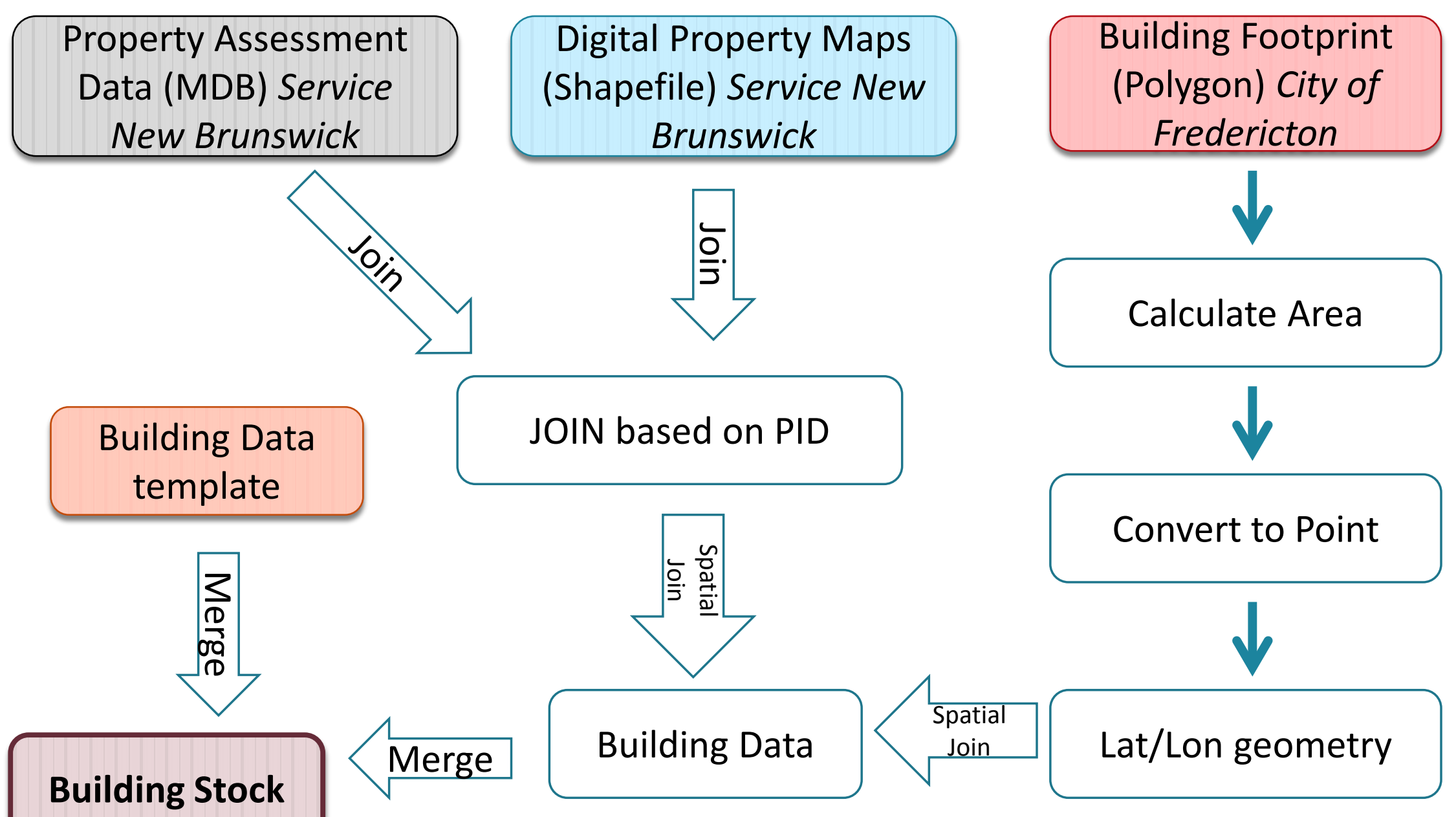




\section{Reclassification}

\begin{tabular}{|c|c|c|c|c|}
\hline Hazus Category & $\begin{array}{c}\text { Hazus } \\
\text { Description }\end{array}$ & NB Code & $\begin{array}{c}\text { NB Code } \\
\text { Description }\end{array}$ & $\begin{array}{c}\text { Property } \\
\text { Description } \\
\text { contains }\end{array}$ \\
\hline RES1 & $\begin{array}{l}\text { Single Family } \\
\text { Home }\end{array}$ & 120 & $\begin{array}{l}\text { Residential } \\
\text { Improved }\end{array}$ & House \& Lot \\
\hline \multirow[t]{2}{*}{ RES2 } & Mobile Home & 104 & $\begin{array}{l}\text { Mobile Home } \\
\text { Parks }\end{array}$ & $\begin{array}{c}\text { Mobile Home } \\
\text { Lots, Pine Grove } \\
\text { LLC }\end{array}$ \\
\hline & & 105 & $\begin{array}{l}\text { Mobile/ Mini } \\
\text { Home w/ Land }\end{array}$ & $\begin{array}{l}\text { Mini Home, } \\
\text { Mobile \& Lot }\end{array}$ \\
\hline RES3A & Duplex 1-2 units & 120 & $\begin{array}{c}\text { Residential } \\
\text { Improved }\end{array}$ & Duplex \\
\hline RES3C & $\begin{array}{l}\text { Apartment } 5-9 \\
\text { units }\end{array}$ & 120 & $\begin{array}{l}\text { Residential } \\
\text { Improved) }\end{array}$ & Apt. Bld \\
\hline COM1 & Retail trade & $\begin{array}{c}202-207,210 \\
214\end{array}$ & $\begin{array}{l}\text { Service stations, } \\
\text { restaurant }\end{array}$ & \\
\hline EDU1 & Grade School & 402 & Schools & EDU1 \\
\hline IND2 & Light industrial & $304-310$ & $\begin{array}{l}\text { Food, Fish, Textile, } \\
\text { Apparel Processing } \\
\text { Plants, Lumber }\end{array}$ & \\
\hline
\end{tabular}




\section{Calculation of Replacement costs}

$$
\begin{aligned}
& \mathbf{V}_{\mathrm{RES} 1, \mathbf{k}}=\left(\mathbf{A}_{\mathrm{RES} 1, \mathbf{k}}\right) *\left[\sum_{i=1}^{4} \sum_{i=j}^{4} w_{i, k} * w_{j, k} * C_{i, j}\right]+ \\
& \left(\mathbf{A}_{\mathrm{RES} 1, \mathbf{k}}\right) * w_{l, k} *\left[\sum_{i=1}^{4} \sum_{i=j}^{4} w_{i, k} w_{j, k} C_{i, j . l}\right]+ \\
& \left(\mathbf{R E S} 1 \mathbf{C n t}_{\mathbf{k}}\right) *\left[\sum_{i=1}^{4} \sum_{i=j}^{4} w_{i, k} w_{m, k} C_{i, m}\right]
\end{aligned}
$$

\begin{tabular}{|c|c|}
\hline $\mathbf{V}_{\text {RES1, }}$ & Total estimated valuation for RES1 for a given census block ( $k$ ) \\
\hline$A_{\text {RES1v,k }}$ & Total RES1 floor area for given (k) \\
\hline $\mathbf{i}$ & Means construction class \\
\hline $\mathbf{w}_{\mathrm{i}, \mathrm{k}}$ & Means construction class weighting factor \\
\hline $\bar{j}$ & Number of stories of RES1 \\
\hline$\overline{w_{i, k}}$ & Means number of stories weighting factor \\
\hline $\mathrm{C}_{\mathrm{ij}}$ & RES1 cost per square foot \\
\hline $\mathrm{I}$ & Basement status (yes [1] or no [2]) \\
\hline $\mathbf{w}_{1, \mathrm{k}}$ & Means basement status weighting factor \\
\hline $\mathrm{C}_{\mathrm{i}, \mathrm{j}, \mathrm{I}}$ & Additional cost per square foot for a finished basement \\
\hline $\mathbf{M}$ & Garage combination (1 car, 2 car, etc.) \\
\hline $\mathbf{w}_{\mathrm{m}, \mathrm{k}}$ & Weighting factor for garage type \\
\hline$c_{i, m}$ & Additional replacement cost for a given garage type \\
\hline$\overline{R_{\text {ES1Cnt }}}$ & Count of RES1 structures within a given census block \\
\hline
\end{tabular}




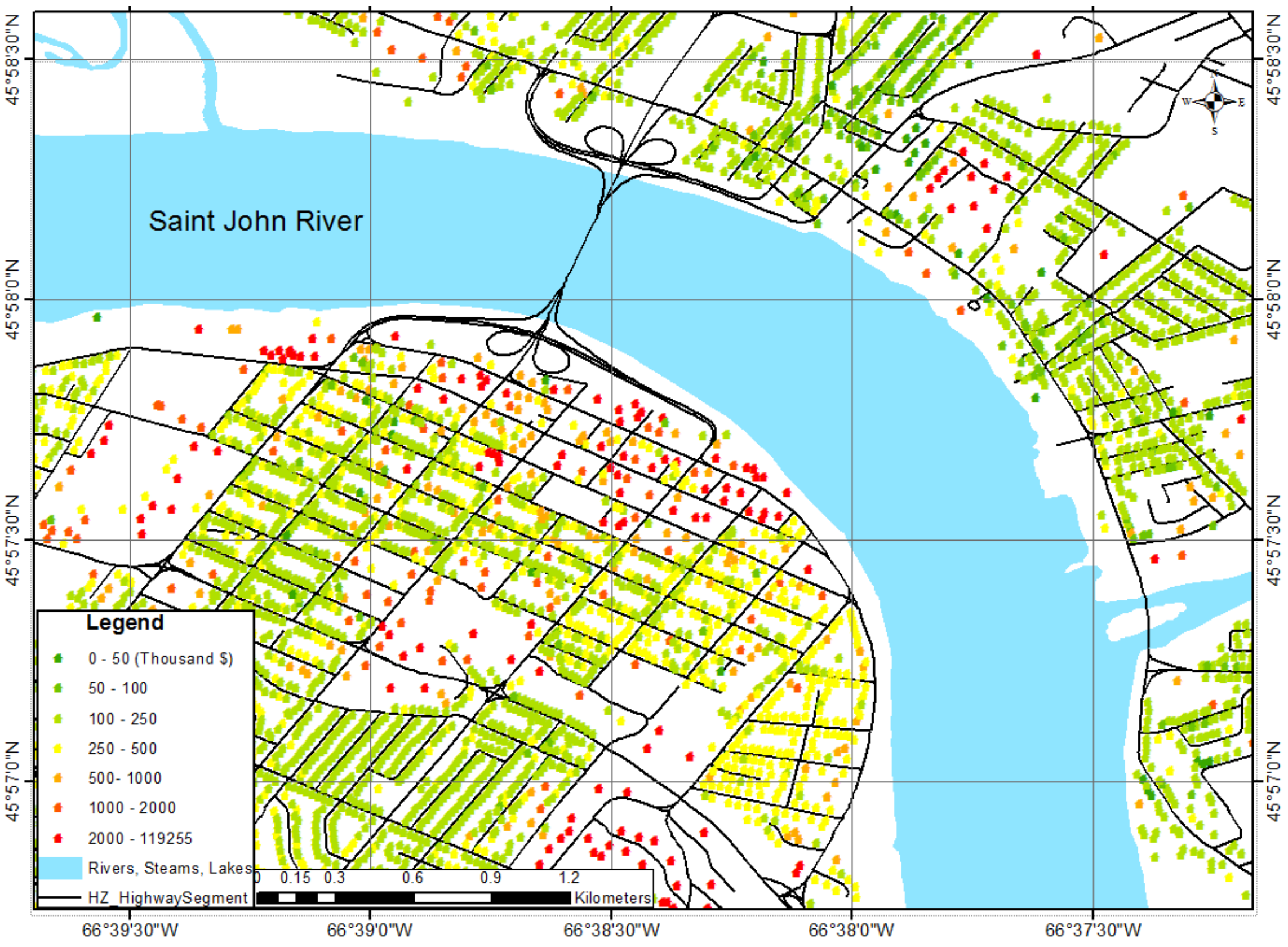




\section{Essential Facilities}

- Hospitals

- Police stations

- Fire Stations

- Schools

- Care Facilities

\section{Schools (txt)}

- Name

- Address

- Contact

- City

- Province

- Comments

- Phone

- Postal Code

*NB Department of Education

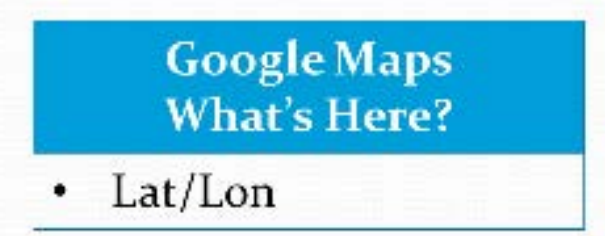

Schools Feature Class

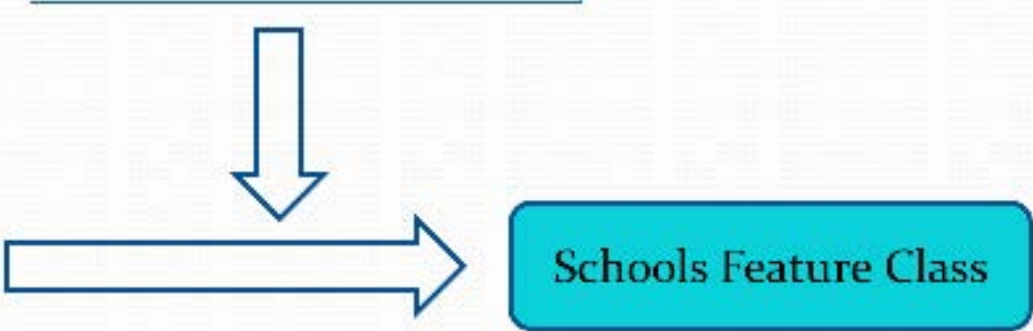

School Data template 


\section{Flood Scenario}

- Flood gauge (Station: 01AK003) indicated the highest water level of $8.359 \mathrm{~m}$ on May 2, 2008

- Flood Stage: $6.50 \mathrm{~m}$

- May 1: 50 roads closed

- Including off ramps at the cities two bridges

- 680 evacuees registered with Canadian Red Cross

- 304 customers without power (May 3)

- Total Cost: \$23 million (Canadian Disaster Database) 


\section{Validation tests}

- Depth damage curves

- Regression equations

- Manning's $n$ values

- Hazus loss estimates vs. real expenditures

- Shelter requirements

- Building damage

- Economic losses
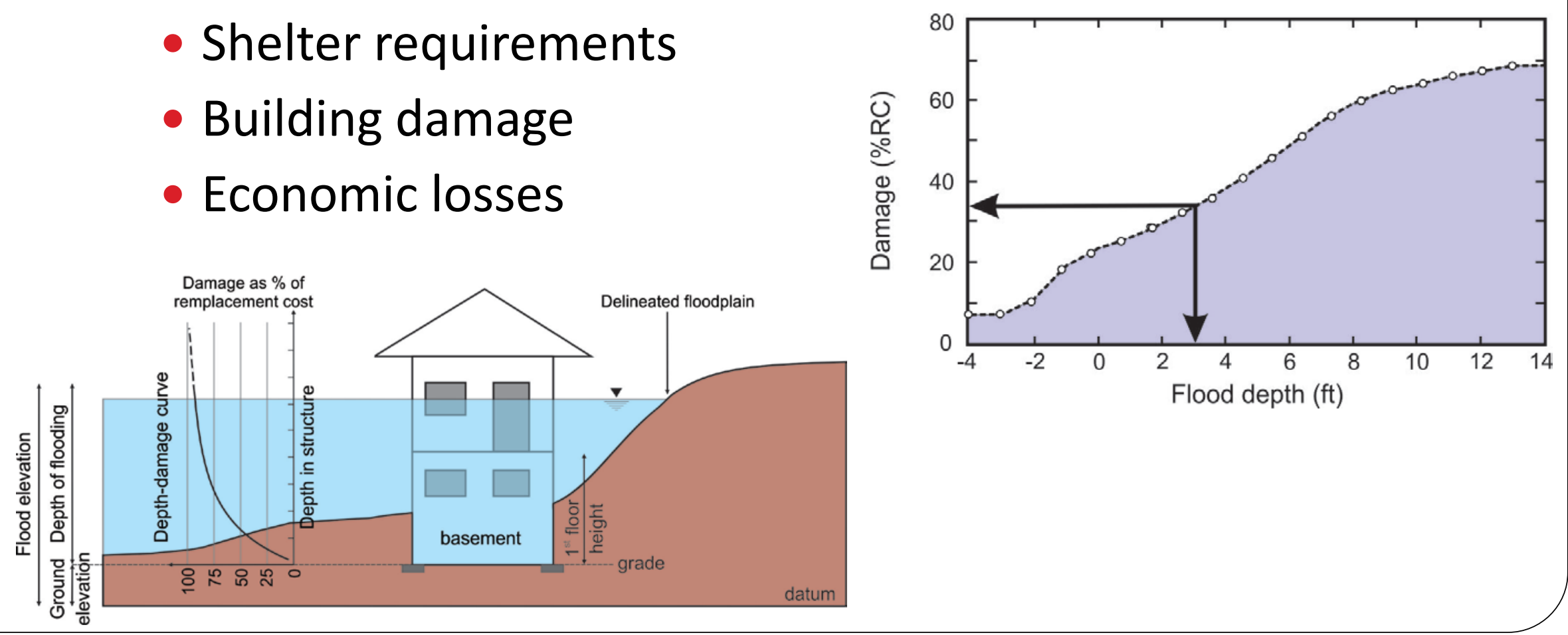


\section{Future Plans}

EvaRISK Interface - Windows Internet Explorer

(3) $>>$ htti://w-stf-a073655/rrat/applet.html

EvaRISK Interface

EvaRISK Damages Casualties

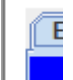

,

100,00

Probability (ㅇ)

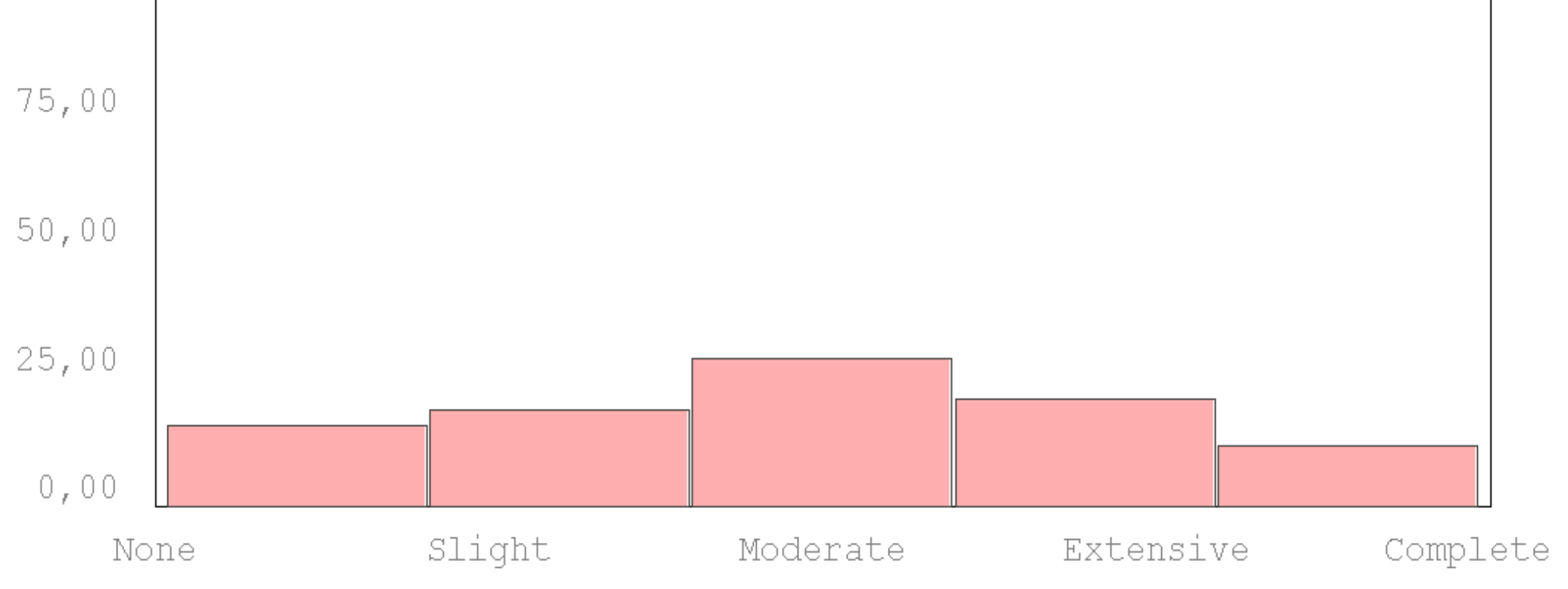

Damage states 

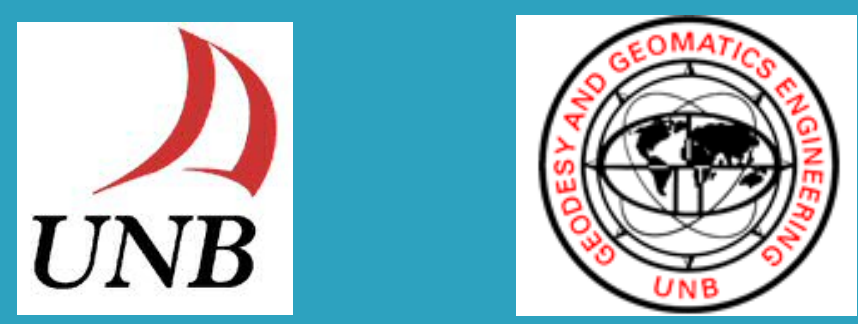

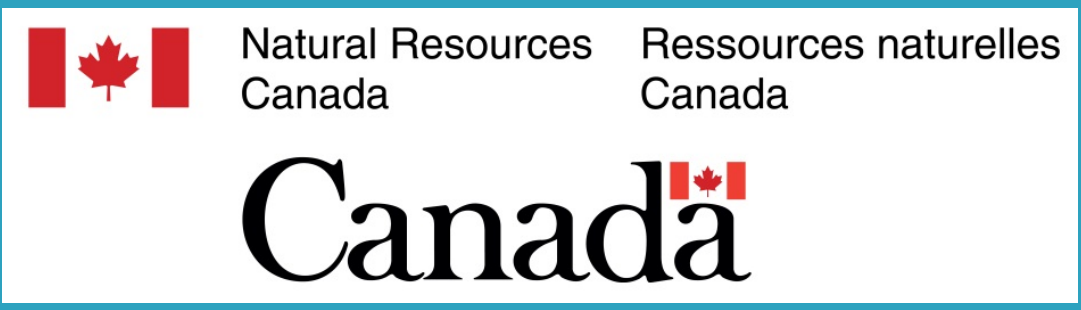

\section{Data Preparation for Validation Study of Hazus Canada Flood Model}

Heather McGrath

Heather.mcgrath@unb.ca 